\title{
Rhines scale and spectra of the $\beta$-plane turbulence with bottom drag
}

\author{
Sergey Danilov ${ }^{1,2}$ and David Gurarie ${ }^{3, *}$ \\ ${ }^{1}$ Institute of Atmospheric Physics, Moscow, Russia \\ ${ }^{2}$ Alfred Wegener Institute for Polar and Marine Research, Bremerhaven, Germany \\ ${ }^{3}$ Case Western Reserve University, Cleveland, Ohio 44106
}

(Received 17 May 2001; revised manuscript received 11 February 2002; published 17 June 2002)

\begin{abstract}
We study two-dimensional incompressible turbulence on the $\beta$ plane and propose a modification to the Rhines scale that takes into account the bottom friction. The modified Rhines scale is studied numerically, and found to predict accurately the jet number and the energy peak of the $\beta$-plane turbulence for strong $\beta$. The intermediate cases show a transition from the (isotropic) friction scale to the Rhines one, as the proper halting scale for the inverse cascade.

DOI: 10.1103/PhysRevE.65.067301

PACS number(s): 47.27.- i, 47.10.+g, 05.40.-a
\end{abstract}

\section{INTRODUCTION}

The peculiar feature of two-dimensional (2D) incompressible turbulence on the $\beta$ plane is the formation of zonal jets, when stirred by various sources, including isotropic smallscale ones. Such behavior was predicted by Rhines [1] who introduced the concept of halting scale $k_{\beta}$ that should divide the $\beta$-plane turbulence into the small-scale isotropic turbulence and highly anisotropic wavelike turbulence at large scales. Rhines sought an explanation for such a transition, in terms of "Rossby wave radiation" by turbulence.

Subsequent development [2-9] clarified the issue. The transformation of "turbulence to waves" should not be taken literally. As the energy is transferred upscale via turbulent cascade, the $\beta$ term in the low-spectral range becomes comparable to nonlinear terms of the vorticity equation. The strong $\beta$ term would require frequency synchronism in that range, and thus make energy transfer less efficient. Hence, the upward cascade would be primarily carried into the region of the small $\beta$ term in the Fourier space, i.e., towards zonal modes $k_{x}=0$. Such spectral trends are clearly seen in simulations of the $\beta$-plane turbulence that use the eddydamped quasi-normal Markovian scheme [4]. In the physical space, the $\beta$ effect leads to the formation of strong zonal jets in the flow.

Rhines defined the transition scale for the upward cascade, due to $\beta=\partial f / \partial y$ (northward gradient of the local Coriolis parameter $f$ ) as

$$
k_{\beta}=\left(\beta / 2 U_{r m s}\right)^{1 / 2},
$$

$U_{r m s}=(2 E)^{1 / 2}$-root mean square (rms) velocity, and $E$ - the total kinetic energy. Other researchers viewed $k_{\beta}$ as an estimate of the energy peak scale $k_{p}$, and the wave number of zonal jets that develop in the turbulent flow $[4,7-10]$.

The Rhines definition involves, however, the undetermined energy content of the flow, which depends on the forcing and dissipation. Only the latter could provide a suitable arrest mechanism at large scales, as dissipation-free

\footnotetext{
*Email address: dxg5@po.cwru.edu
}

flows (with or without $\beta$ ) keep accumulating energy at the gravest modes [4]. So, the $\beta$ term alone could not halt the cascade.

The natural dissipation mechanism in physical systems is bottom (Rayleigh) friction, which affects all scales equally. Computationally, one often uses scale-selective hypofriction, which suppresses only the gravest modes, which hence could recreate an inertial interval between forcing and dissipation. Clearly, turbulent flows stabilized by the friction have their energy content, hence rms velocity and the Rhines scale, determined by the balance between forcing and dissipation.

The (approximate) energy equation, which accounts for the bottom friction with the coefficient $\lambda$ and sources $\varepsilon$ (injection rate) but drops other (viscous) losses,

$$
\partial_{t} E \approx-2 \lambda E+\varepsilon,
$$

yields the equilibrium energy level $E_{e} \approx \varepsilon / 2 \lambda$.

In numerical simulations the energy injection rate remains nearly constant for small-scale Gaussian and short-correlated non-Gaussian sources, so one could estimate the Rhines scale by

$$
k_{\beta}=(\beta / 2)^{1 / 2} \lambda^{1 / 4} \varepsilon^{-1 / 4} .
$$

Our goal here is to verify numerically such an estimate and its relation to the observed number of zonal jets in the flow, and its energy peak.

Scale (2) combines friction with $\beta$. We shall discuss the role of both in arresting the inverse cascade, and compare Eq. (2) to the friction scale of the "isotropic turbulence" $(\beta=0)$, determined by the balance of forcing and dissipation

$$
k_{f r}=\left(3 C_{K}\right)^{3 / 2}\left(\lambda^{3} / \varepsilon\right)^{1 / 2} .
$$

Relation (3) assumes the standard $-5 / 3$ spectrum typical of the isotropic turbulence with Kolmogorov constant $C_{K}$.

To examine the role of friction in the $\beta$-plane turbulence we conducted a series of experiments on $512^{2}$ lattice $(2 \pi$ $\times 2 \pi$ computational domain), using a dealiased pseudospectral code [12], with the third-order Adams-Bathforth time stepping. The standard vorticity equation for $\zeta=\Delta \psi(\psi$ is the stream function) with dissipation $D$ and forcing $f$,

$$
\partial_{t} \zeta+\left(\partial_{x} \psi \partial_{y} \zeta-\partial_{y} \psi \partial_{x} \zeta\right)+\beta \partial_{x} \psi=D[\zeta]+f,
$$


TABLE I. Basic parameters of numeric experiments. The frictional scale is computed via $k_{f r}=50\left(\lambda^{3} / \varepsilon\right)^{1 / 2}[11]$.

\begin{tabular}{cccccccc}
\hline \hline Run & $\lambda$ & $\beta$ & $k_{j e t} / k_{\beta}$ & $k_{p} / k_{\beta}$ & $k_{\beta} / k_{f r}$ & $E_{z} / E$ & $k_{\beta}$ \\
\hline 1 & 0.03 & 20 & 1.0 & 1.1 & 0.96 & 0.03 & 7.0 \\
2 & 0.03 & 20 & 1.0 & 1.0 & 1.1 & 0.06 & 6.0 \\
3 & 0.03 & 40 & 0.72 & 0.83 & 1.6 & 0.13 & 8.4 \\
4 & 0.03 & 80 & 0.84 & 0.84 & 2.3 & 0.20 & 11.9 \\
5 & 0.03 & 160 & 0.78 & 0.83 & 3.2 & 0.27 & 16.8 \\
6 & 0.03 & 320 & 0.88 & 0.88 & 4.5 & 0.42 & 23.8 \\
7 & 0.03 & 640 & 0.79 & 0.70 & 6.5 & 0.41 & 33.1 \\
8 & 0.01 & 40 & 0.75 & 0.75 & 6.0 & 0.32 & 6.6 \\
9 & 0.01 & 80 & 0.76 & 0.65 & 8.7 & 0.36 & 9.2 \\
10 & 0.01 & 160 & 1.0 & 0.63 & 12.7 & 0.35 & 12.6 \\
11 & 0.01 & 640 & 0.75 & 0.75 & 25.3 & 0.55 & 25.3 \\
12 & 0.1 & 640 & 0.76 & 0.76 & 1.4 & 0.20 & 45.0 \\
\hline \hline
\end{tabular}

is expanded in Fourier modes $\mathbf{k}=\left(k_{x}, k_{y}\right)$

$$
\partial_{t} \zeta_{\mathbf{k}}+J_{\mathbf{k}}+i \beta k_{x} \psi_{\mathbf{k}}=-D_{\mathbf{k}} \zeta_{\mathbf{k}}+f_{\mathbf{k}} .
$$

The dissipation operator in our simulations combines hyperviscosity (at high $k$ ) with the bottom drag $D_{\mathbf{k}}=\lambda$ $+\nu\left(k / k_{\max }\right)^{2 n}$.

The source is given by a narrow-band Markov process in the wave-number range $\left(k_{f}-2, k_{f}+2\right)$ centered around the forcing scale $k_{f}$. So, $f_{\mathbf{k}}(t+\delta t)=A\left(1-r^{2}\right)^{1 / 2} e^{i \theta}+r f_{\mathbf{k}}(t)$, where $A$ is the source amplitude, $r$ defines its correlation radius $\delta t /(1-r)$, and $\theta$-random uniformly distributed phase on $[0,2 \pi]$. Despite its non-Gaussian form, we found the energy injection rate $\varepsilon$ by such a source to be independent of $\beta$ and $\lambda$ and to remain nearly stationary.

We conducted a series of 12 experiments with parameters $k_{f}=150$ and $n=8$ in the first run, and lower values $k_{f}$ $=100$ and $n=4$ for the rest of them. Table I summarizes parameters $\beta, \lambda$ of different runs, ratios of the jet wave number $k_{j e t}$ and the energy-peak wave number $k_{p}$ to $k_{\beta}$, ratio $k_{\beta} / k_{f r}$, the ratio of zonal to total energy $E_{z} / E$, and the scale $k_{\beta}$.

We have chosen our basic parameters such that $k_{\beta}$ will span a sufficient range of wave numbers (within the limits of resolution). Since scaling (2) is more sensitive to $\beta$ than $\lambda$ or the energy flux, we mostly varied $\beta$. Our experiments also cover a range of parameter $k_{\beta} / k_{f r}$.

The turbulent $\beta$-plane flow takes a long time $O(1 / \lambda)$ to equilibrate. Its zonal component evolves even more slowly. So, we carried out integration in runs 4 and 7 to time $t$ $=9 / \lambda$, and in run 12 to $t=30 / \lambda$. In all three cases we attained a near-equilibrium value of the zonal energy. We observed the energy peak of the zonal flow to stabilize after $t$ $=3 / \lambda$, so all other runs were integrated to $t=6 / \lambda$. All runs but the first one had approximately equal energy injection rates $\varepsilon \approx 0.0025$, while the first one had about half that rate.

\section{RESULTS}

Figure 1 plots the experimentally measured jet number $k_{j e t}$ (triangles) and energy peak $k_{p}$ (pluses) versus $k_{\beta}(2)$. We

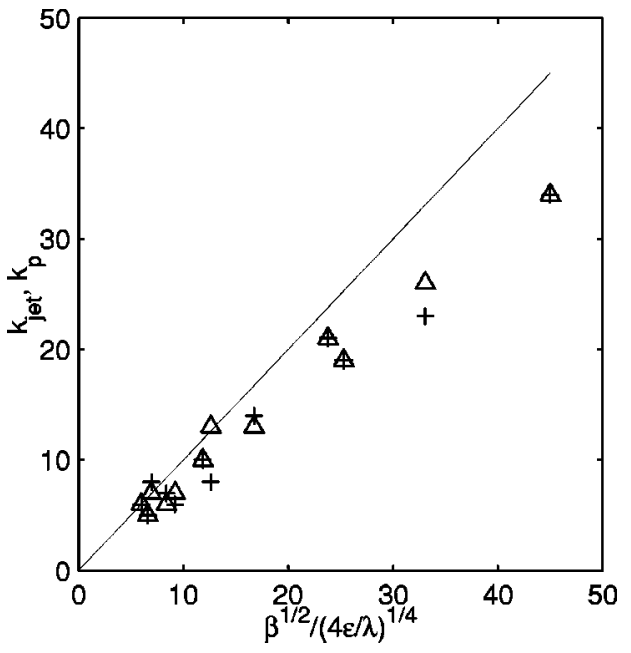

FIG. 1. Measured $k_{j e t}(\Delta)$ and $k_{p}(+)$ vs $k_{\beta}(2)$ in all experiments.

identified $k_{\text {jet }}$ with the energy peak on the zonal $\left(k_{x}=0\right)$ axis of the $\mathbf{k}$ plane, and $k_{p}$ with the isotropic energy peak. Two numbers come close, and often coincide in flows dominated by strong zonal jets. Although ratios $k_{j e t} / k_{\beta}$ and $k_{p} / k_{\beta}$ vary from one experiment to the other (see Table I), they remain close to 1 , which confirms scaling (2). The jet number $k_{j e t}$ varies from 5 in experiment 8 to 34 in experiment 12 . The larger values (close to the forcing scale) would correspond to poorly resolved jets in the physical space.

Next we shall discuss the relation between two scales $k_{\beta}$ and $k_{f r}$ and their role in the arrest mechanism. While the bottom drag alone could arrest the inverse cascade with or without $\beta$, the latter serves only to redistribute energy in the Fourier space by channeling it into the zonal directions [1].

Such redistribution becomes impossible for low $\beta$, when $k_{\beta} \leqslant k_{f r}$. Such flows could not develop organized jets, and stay nearly isotropic. We demonstrate this in Fig. 2, which shows isotropic energy spectra $E(k)$ and zonal spectra $E_{z}(k)$ for experiments 2, 5, and 7 at the final (stable) phase of their evolution. These experiments have equal forcing and dissipation, but different $\beta(20,160$, and 640). Hence, they share identical $k_{f r}$ but different $k_{\beta}$.

The isotropic spectrum of experiment 2 obeys the $-5 / 3$ law almost everywhere in the energy range except for a small bulge close to $k_{p}$. Here, the zonal component of the energy spectrum is relatively low, and its peak at $k=k_{j e t}$ carries only a fraction of the bulge's energy. Physical space realizations of the vorticity field have barely visible zonal bands (not shown), revealed only in the zonal mean flow.

The ratio $k_{f r}$ to $k_{\beta}$ (see Table I) is close to 1 in experiment 2 , making it a marginal case for the zonal structure. Further decrease of $\beta$ would destroy zonal flow altogether.

Well-organized jets typically appear for $k_{\beta}>k_{f r}$ (all experiments except 1,2 , and 12). Here, the energy spectrum becomes highly anisotropic and deviates noticeably from the standard $k^{-5 / 3}$ scaling in the vicinity of $k_{p}$ (bulge). The spectra in Fig. 2 illustrate the development of anisotropy and the departure from the $k^{-5 / 3}$ as $\beta$ increases. The middle pair 


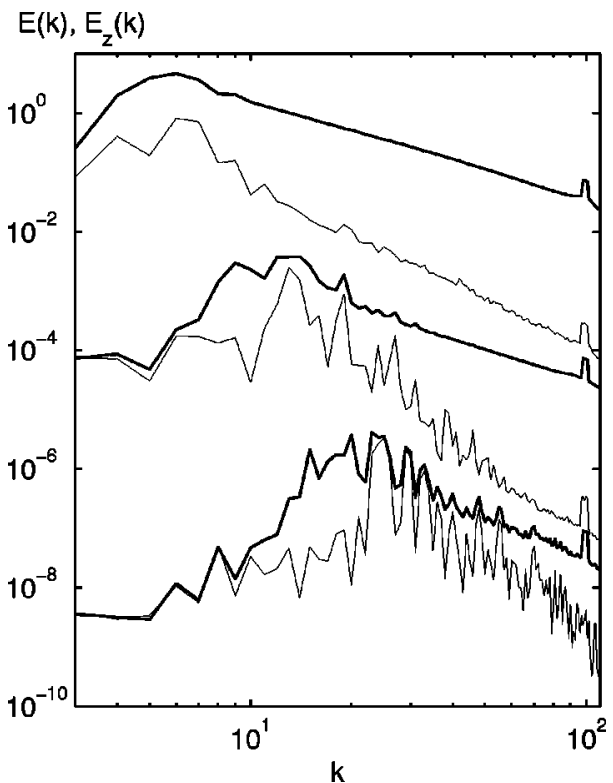

FIG. 2. Isotropic (thick) and zonal (thin) energy spectra in experiments 2 (top pair), 5 (middle pair), and 7 (bottom pair). The top and bottom pairs are shifted by three orders to top and to bottom, respectively.

(experiment 5) shows two peaks between $k=10$ and 20 in the isotropic spectrum coming from its zonal component. Experiment 7 shows even greater departure from the standard $k^{-5 / 3}$ isotropic scaling, due to a stronger zonal contribution.

Consequently, estimate (3) of $k_{f r}$, based on the $5 / 3$ assumption, loses its predictive power. As the bulk of the energy accumulates and dissipates at $k_{\beta}$, the latter takes on the role of the halting scale. Let us stress, however, that such $k_{\beta}>k_{f r}$ acquires its "halting status" only through the combined effect of the $\beta$ and friction. The largest of two estimates (3) and (2) could determine the arrest scale of 2D turbulence for any $\beta$, small or large. This agrees with estimates in the recent paper [10], which argues that the Rhines and actual frictional scales coincide if the zonal energy spectrum has a slope of -5 .

The energy-peak wave number $k_{p}$ of the isotropic spectrum coincides with $k_{j e t}$ in some experiments, but could be lower in general (see Table I). One gets a significant "nonzonal" input near the energy peak, due to so-called satellite modes, in the cusp region of the $2 \mathrm{D}$ energy spectrum. In most cases, we observed strong satellites with small $k_{x}$ (one to several units) and $\left|k_{y}\right|$ close to $k_{j e t}$, containing as much energy as (and probably more than) the gravest zonal modes. Thus, experiment 4 shows the local maximum of the 2D energy spectrum at $\left(\left|k_{x}\right|,\left|k_{y}\right|\right)(1,8)$, with an amplitude only 1.3 times less than the maximal zonal amplitude at $(0,10)$. This mode contained $14 \%$ of the total energy, while the entire zonal spectrum carried $20 \%$. Experiment 7 gave $7.2 \%$ of the total energy residing at the zonal peak $(0,25)$, and $7.9 \%$ at the satellite $(1,20)$.

The presence of strong satellites explains the difference between $k_{p}$ and $k_{j e t}$ in our experiments. In the physical space, satellites modulate zonal jets in the $x$ direction.

Although frictional scale $k_{f r}$ (3) does not apply directly to the $\beta$-plane turbulence in the strong zonal regime $\left(k_{f r}\right.$ $<k_{\beta}$ ), the ratio $k_{\beta} / k_{f r}$ (with properly adjusted Kolmogorov constant) could serve as a qualitative measure of anisotropization of such flows. Indeed, the energy that would otherwise fill the isotropic " $5 / 3$-background" range between $k_{f r}$ and $k_{\beta}$ is now piling up at $k=k_{\beta}$ in the predominantly zonal or satellite modes. So, a large ratio would indicate anisotropy with a significant share of zonal energy. We illustrate it in column 7 of Table I, which gives the ratio of zonal over total energy in all experiments.

\section{ACKNOWLEDGMENTS}

This work was sponsored by the GTP program at NCAR. The first author was supported by RFFI, Grant Nos. 99-0564350 and 99-05-64351, and an NRC/NAS Cobase Grant. We thank V. Gryanik and J. Herring for stimulating discussions.
[1] P.B. Rhines, J. Fluid Mech. 69, 417 (1975).

[2] G. Holloway and M. Hendershott, J. Fluid Mech. 82, 747 (1977).

[3] M.E. Maltrud and G.K. Vallis, J. Fluid Mech. 228, 321 (1991).

[4] G.K. Vallis and M.E. Maltrud, J. Phys. Oceanogr. 23, 1346 (1993).

[5] T. Nozawa and S. Yoden, Phys. Fluids 9, 2081 (1997).

[6] J.Y.-K. Cho and L.M. Polvani, Phys. Fluids 8, 1531 (1996).

[7] H.-P. Huang and W.A. Robinson, J. Atmos. Sci. 55, 611 (1998).
[8] A. Chekhlov, S.A. Orszag, S. Sukoriansky, B. Galperin, and I. Staroselsky, Physica D 98, 321 (1996).

[9] H.-P. Huang, B. Galperin, and S. Sukoriansky, Phys. Fluids 13, 225 (2001)

[10] B. Galperin, S. Sukoriansky, and H.-P. Huang, Phys. Fluids 13, 1545 (2001).

[11] S. Danilov and D. Gurarie, Phys. Rev. E 63, 020203(R) (2001).

[12] G.S. Patterson and S.A. Orszag, Phys. Fluids 14, 2538 (1971). 